\title{
Rules of Emotions: A Linguistic Interpretation of an Emotion Model for Affect Sensing from Texts
}

\author{
Mostafa Al Masum Shaikh ${ }^{1}$, Helmut Prendinger ${ }^{2}$, and Ishizuka Mitsuru ${ }^{1}$ \\ ${ }^{1}$ Department of Information and Communication Engineering, University of Tokyo, \\ 7-3-1 Hongo, Bunkyo-ku, 113-8656 Tokyo, Japan \\ mostafa@mi.ci.i.u-tokyo.ac.jp, ishizuka@i.u-tokyo.ac.jp \\ ${ }^{2}$ Digital Contents and Media Sciences Research Division, National Institute of Informatics, \\ 2-1-2 Hitotsubashi, Chiyoda-ku, 101-8430 Tokyo, Japan \\ helmut@nii.ac.jp
}

\begin{abstract}
This paper aims to interpret the cognitive theory of emotions known as the OCC emotion model from the computational linguistic standpoint. Since the OCC emotions are associated with several cognitive variables, in this paper we explain how the values could be assigned to those by analyzing and processing natural language components.
\end{abstract}

\section{Characterization of the OCC Emotions}

There are two kinds of variables, namely, emotion inducing variables (event, agent and object based) and emotion intensity variables. The event-based variables are usually calculated with respect to the event which is usually a verb-object pair found in the sentence. For example, the sentence, John bought Mary an ice-cream, gives an event as (buy, ice-cream).

The OCC emotion model specifies 22 emotion types and 2 cognitive states. The cognitive and appraisal structure of the OCC emotion types can be characterized by some rules interplaying with several variables. For example, literal definitions of "Happy-for" and "Fear" emotion are respectively, "Pleased about an event Desirable for a Liked agent" and "Displeased about Negative Prospect of an Undesirable Unconfirmed event".

\section{Assigning Values to the Variables}

The values for the variables self_presumption $(s p)$ and self_reaction $(s r)$ are set "Desirable" or "Undesirable", and "Pleased" or "Displeased" towards an event if the valence of that event is positive or negative, respectively. In the example above, the event "buy ice-cream" gets a positive valence $(+8.433)$, and hence the values for $s p$ and $s r$ for this event are "Desirable" and "Pleased", respectively. But, for the sentence "A terrorist escaped from the Jail" the value for other_presumption (op) for the event "escape jail" is presumably "Desirable" for the agent "terrorist" but it is usually "Undesirable" and "Displeased" for $s p$ and $s r$, respectively. Hence, the value for $o p$ is set "Undesirable" if an event of negative or positive valence is associated with an agent/object having positive (e.g., the police was sacked) or negative (e.g., the 
criminal was taken to trial) valence respectively. The value for cognitive_strength (cs) indicates how closely the program considers selfness. This value is set as "Self" if the agent described in the text is a first person (i.e., $I$ or We); otherwise it is set as "Other". If the tense of the verb is present or future, the value for status (stat) is set as "Unconfirmed" (e.g., I am trying to solve it.); and if it is past or modal without a negation, stat is set "Confirmed" (e.g., I succeeded.), but if with a negation, stat is set "Disconfirmed" (e.g., I did not succeed.). If the valence of the agent/object is positive, "Like" is set for $a f$ and of variables. The value for self_appraisal $(s a)$ is set "Praiseworthy" if a positive value or zero is assigned for the event; otherwise "Blameworthy" is set. The value of object_appealing (oa) indicates whether an object is "Attractive" or "Unattractive". "Attractive" is set if the object has a positive valence with a familiarity score higher than a threshold value. The familiarity score is obtained from the ConceptNet by calculating the percentage of nodes (out of 300000 concept-nodes) linking the given object/concept. For example, the familiarity score for "restaurant" is $0.4872 \%$. The value for valenced_reaction ( $v r)$ is obtained from the SenseNet's valence analysis. We consider $v r$ to be "True" if the sentiment-valence become above than 4 or less than -4 . The value for intensity variable event_deservingness $(e d)$ is "High" for an event having positive valence or "Low" for negative one. If an action is qualified with an adverb (e.g., He worked very hard.) or target object qualified with an adjective (e.g., I am looking for a quiet place.) without a negation the value for effort_of_action (eoa) is set "Obvious". The variable expected_deviation (edev) indicates the difference between the event and its actor. For example, in the sentence "The police caught the criminal finally.", the actor "police" and the event "catch criminal" don't deviate because the action is presumably expected by the actor. We set the value for edev as "Low" if ConceptNet returns any semantic relationship between the actor and event. The values "Common" or "Uncommon" are set for event_familiarity (ef) according to the familiarity score obtained from ConceptNet for the event.

For example, the sentence "I suddenly got to know that my paper won the best paper award.”, may output the following sets of emotions: \{Joy, Satisfaction, Surprise, Pride, Gratification $\}$. In the example the event, "win award" and object "best paper award" set the values for $c s$ to "Self", of to "Like", $s r$ to "Pleased", sp to "Desirable", pros to "Positive", stat to "Confirmed", unexp to true (because of token 'suddenly') and $v r$ to "True". In order to reduce the number of emotions we can consider the intensity variables and thus we can output \{ Satisfaction, Surprise, Pride $\}$ for this example sentence because "Joy" doesn't have any intensity variable, but on the contrary the intensity variables $e d$ and $e d e v$ are set to "High".

\section{Reference}

1. Ortony, A., Clore, G.L., Collins, A.: The Cognitive Structure of Emotions. Cambridge University Press, Cambridge (1988) 